\title{
Comparison of three different instruments for orthodontic study model analysis
}

SADJ July 2020, Vol. 75 No. 6 p298 - p302

JC Julyan ${ }^{1}$, J Julyan², J de Lange ${ }^{3}$

\section{ABSTRACT}

\section{Introduction}

A proper model analysis forms a vital part of the orthodontic diagnosis process, but it remains a timeconsuming procedure. In day-to-day practice, many orthodontists assess the models subjectively, without applying analytical tests, due to the time it takes to do proper model analysis. ${ }^{1,2}$

Plaster dental models have long been the gold standard for orthodontic study model analysis and to calculate the Bolton index for tooth size disproportions, as well as intra-arch space discrepancies.,3 Vernier callipers or needle pointed dividers are traditionally used to perform measurements on dental models. ${ }^{5}$ More recently digital orthodontic study models that are computer-based have been developed and have the potential to replace the traditional plaster orthodontic models. ${ }^{6}$

\section{Aims and objectives}

The aim of this study was to do model analysis on one hundred orthodontic cases by making use of three different measuring tools. The objective was to see if a difference exists with regards to the measurements produced by the three different instruments and to compare the instruments with each other.

\section{Material and Methods}

Three different instruments were used to measure five values on one hundred orthodontic study models. The three instruments included a Boley Gauge, Digital Vernier Calliper and Carestream 3600 scanner with accompanying software.

\section{Author affiliations:}

1. JC Julyan: $B C h D(U P), P D D$ (UWC), MSc (UWC), General Dentist Private Practice.

ORCID Number: 0000-0002-6186-5724

2. Jennifer Julyan: $B C h D(U P), P D D$ (UWC), Prosthodontic registrar, University of the Western Cape, South Africa.

ORCID Number: 0000-0002-9347-5341

3. Johny de Lange: Bcomm Law (NWU), BChD (UP), MBChB (UP), DipOral Surg (CMSA), DipDent (UP), Medical Intern George Hospital, Western Cape, South Africa.

ORCID Number: 0000-0002-4061-5267

Corresponding author: JC Julyan

General Dentist Private Practice, 40 Wellington Road, Durbanville, Cape Town, 7550.

Email: jcjulyan@gmail.com

Author contributions:

1. JC Julyan: Principal researcher and author of manuscript - $70 \%$

2. Jennifer Julyan: Co-author $-20 \%$

3. Johny de Lange: Co-author - $10 \%$
The five values measured on the study models were: maxillary intercanine width, maxillary intermolar width, mesio-distal width of tooth 11, mesio-distal width of tooth 46 and mesio-distal width of tooth 41 .

\section{Results}

The statistical analysis performed showed that the difference in measurements produced by the three instruments were not statistically significant for the inter-molar width $(p=0.849)$, intercanine width $(p=0.657)$, mesiodistal width of tooth $11(p=0.178)$ and mesio-distal width of tooth $41(p=0.240)$.

The difference in measurements for the mesio-distal width of tooth 46 were statistically significant $(p<0.01)$. However no clinically significant difference was found when the measurements produced by the three instruments were compared.

\section{Conclusions}

All three of the instruments produced accurate measurements and can be used confidently when doing a comprehensive study model analysis for orthodontic diagnosis and treatment planning. The values produced were similar for all three instruments with insignificant differences between the three.

\section{INTRODUCTION}

Successful orthodontic treatment requires a comprehensive diagnosis and treatment plan. Some of the fundamental factors of the diagnosis include: space analysis, arch form, tooth sizes and tooth-arch discrepancies. ${ }^{7}$

A comprehensive model analysis is a vital part in the orthodontic diagnostic process and should always be included. Orthodontic study models are used to plan treatment and to determine the extent of space deficiency or tooth material discrepancy. A conventional model analysis consists of measuring the arch form, width and length as well as the intercanine and intermolar width.

Conventional plaster orthodontic study models have long been proven to be the gold standard for diagnosis and treatment planning in orthodontics. The plaster models also have the advantage of being inexpensive.

Their use has recently started to decline due to intra-oral scanners that can produce digital models. Begole ${ }^{8}$ was one of the first authors introducing a computer program to aid the direct analysis of study models. 
Rudge $^{9}$ devised another computer system using an electronic $X-Y$ reader in order to relate changes in dentition as a result of orthodontic treatment. At the same time, Yen ${ }^{10}$, proposed a simple computer program using a study model photocopy. This program predicts "required space" and compares it to "available space".

OrthoCAD (Cadent, Carlstadt, NJ) developed virtual digital dental casts in the late 1999. Soon after in $2001 \mathrm{E}$ models (GeoDigm, Chanhassen, Minn) developed their own version of digital dental casts. The technology developed by these two companies enabled orthodontists to send alginate impressions to these companies for the fabrication of a 3-dimensional (3D) computerized image.

These 3D images could then be accessed by the orthodontist and used for viewing and planning treatment of patients. ${ }^{3}$

The replacement of conventional plaster orthodontic study models with digital models can benefit orthodontists in the following ways:

1. Models can be accessible instantly on a computer screen without having to retrieve them from storage.

2. Save money on storage costs and laboratory fees.

3. Accurate measurement of tooth and arch sizes, and severity of malocclusion.

4. The ability to send the file containing the digital models anywhere in the world for consultation with colleagues. $^{3}$

Digital models constructed by an intra-oral scanner do not require impression material or plaster of paris and can therefore be used to evaluate the changes after orthodontic treatment without the added laboratory costs and time that it takes to construct orthodontic study models. Most of the normal parameters on the digital models can be measured reliably, and the digital models can be used to eventually eliminate the requirement for producing and storing multiple dental casts.

The digital orthodontic models have been found to be as reliable as traditional stone models and will probably become the standard for orthodontic clinical use in the near future. ${ }^{11}$ When doing model analysis on conventional plaster of paris orthodontic study models, instruments like the Boley gauge and digital vernier callipers are commonly used. Measurements made by callipers are regarded as the gold standard against which other techniques are compared for accuracy. ${ }^{6}$

Various studies have been done to compare measurements made on conventional plaster models and digital models. Studies done by Zilberman et al. ${ }^{12}$ and Garino and Garino ${ }^{13}$ compared linear measurements obtained from conventional plaster and digital models and found a statistically significant difference when comparing the two types of models. They did however conclude that although statistically there was a difference, it was clinically insignificant. Tomassetti et al. ${ }^{14}$ carried out Bolton analyses on digital and plaster models.
He made use of a Vernier calliper on the plaster models and software for the digital models. No statistical significant difference was found between the two different types of models. According Hirogaki et al. ${ }^{15}$, Santoro et al. $^{16}$, Quimby et al. $^{17}$, the use of computer based digital orthodontic study models possess the potential to replace the conventional plaster orthodontic study models.

The objective of this study was to compare measurements made on orthodontic study models by three available instruments for orthodontic study model analysis.

The measurements using the Boley guage and digital Vernier calliper were done using the same plaster orthodontic models and the digital version of the same models were measured using Carestream model software after the models were digitized using a Care Stream CS 3600 intra-oral scanner.

\section{MATERIALS AND METHODS}

The study was conducted in a private practice in Cape Town. The sample comprised of one hundred plaster orthodontic study models of treated patients. All the study models were of good quality and included Class I, II and III malocclusions. All models had fully erupted permanent teeth including incisors, canines, premolars and first molars.

The morphology of the teeth were normal without any attrition, caries, fractures or restorations affecting the measurements. The gender distribution of the 100 cases used included 65 females and 35 males. The average age of all the cases were 15 years and 11 months.

Five parameters were measured on all the orthodontic study models using the three different techniques and then compared with each other.

These parameters included:

1. Maxillary intercanine width (tooth $13-23$ )

2. Maxillary intermolar width (tooth $16-26$ )

\section{Mesio-distal width of tooth 11}

\section{Mesio-distal width of tooth 46}

\section{Mesio-distal width of tooth 41}

The Boley gauge (Figure 1) and digital vernier calliper (Figure 2) were used to measure the plaster models directly by making use of points standardized for all cases.

The same one hundred study models were then scanned and measured using software provided by the Carestream CS 3600 scanner (Figure 3)

All one hundred cases were marked on the mesiopalatal cusps of teeth 16 and 26, as well as the cusp tips of teeth 13 and 23 (Figure 4). These markings provided standardized reference points to use during the measuring process. 


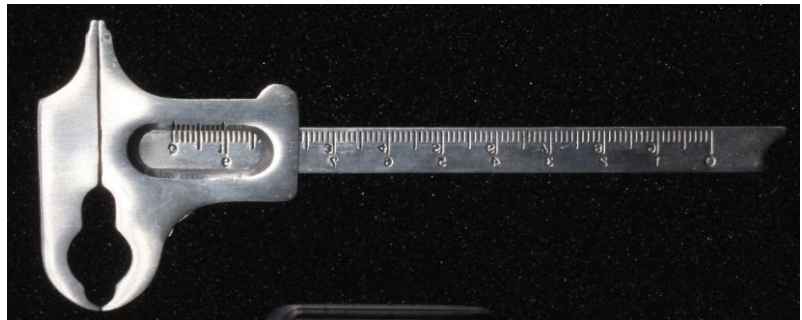

Figure 1. Boley gauge.

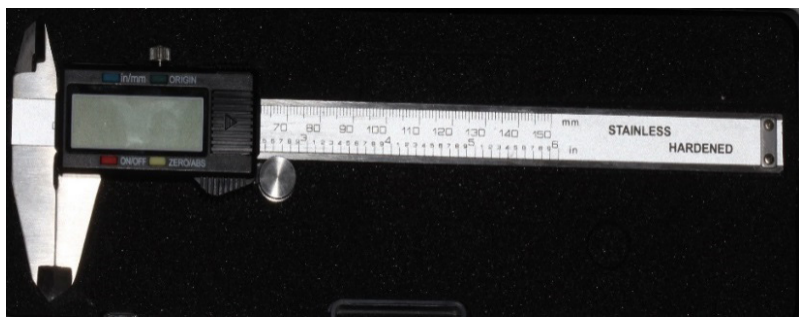

Figure 2. Digital Vernier calliper

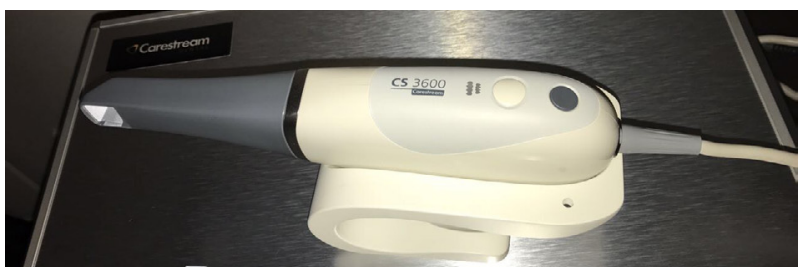

Figure 3. Carestream CS3600 intra-oral scanner
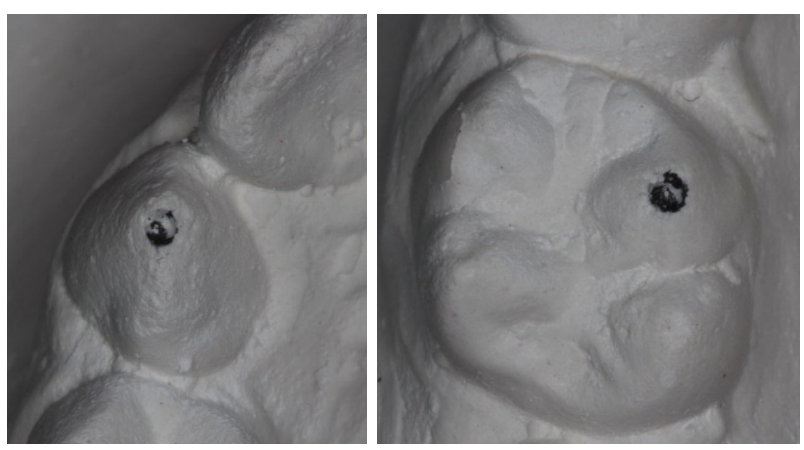

Figure 4. Canine and molar marked with diamond bur.

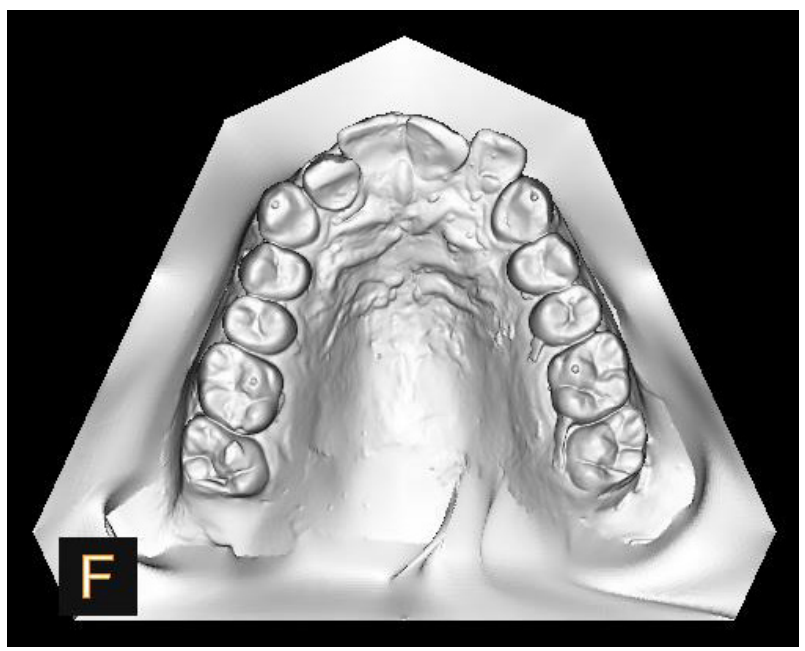

Figure 5. Example of how the standardised markings were used in order to measure on the digital orthodontic study models produced by the intra-ora scanner.
The markings were made using a small round diamond bur. The mesio-distal widths of teeth 11,46 and 41 were measured at the greatest mesio-distal dimension between the two contact points.

The scans produced by the intra-oral scanner clearly showed the indentations made by the bur and were also used for standardizing the measurements, see Figure 5. The digital models were obtained by scanning the plaster orthodontic study models using a Carestream intra-oral scanner and imported as STL (Standard Tessellation Language) files into the Carestream model software where they could be rotated and magnified to help facilitate the measuring process.

All the measurements were done by the author of this article. All one hundred cases were measured and documented by the same operator. All the values were placed in an Excel file in order to conduct the statistical analysis of all the measurements and to compare the three different techniques with one another. The five measurements of all the cases were compared.

For the statistical analysis the three instruments were treated as independent variables. The difference between the instruments were tested per aspect in an attempt to avoid variation between the aspects and possible differences in measurement units. Models were treated as a co-variate in an attempt to control unexplained variation between the models.

\section{RESULTS}

The results for the five parameters measured by each of the three instruments are summarized below:

The difference between the instruments with intermolar as dependent variable was not significant: $F_{2,296}=0.163$; $p=0.849$, see Table 1 .

The difference between the instruments with intercanine as dependent variable was not significant: $F_{2,296}=0.421$; $\mathrm{p}=0.657$, see Table 2 .

The difference between the instruments with the mesiodistal width of tooth 11 as dependent variable was not significant: $F_{2,296}=1.735 ; p=0.178$, see Table 3 .

The difference between the instruments with the mesio-distal width of tooth 46 as dependent variable was significant: $F_{2,296}=7.097 ; p<0.01$, see Table 4 .

The difference between the instruments with the mesiodistal width of tooth 41 as dependent variable was not significant: $F_{2,296}=1.434 ; p=0.240$, see Table 5 .

\section{DISCUSSION}

Orthodontic study models are used routinely for model analysis and treatment planning. The use of digital study models provides the opportunity to accurately determine the effects of orthodontic treatment and to do comprehensive model analysis without the need for impressions and storage. The purpose of the study was to evaluate if a difference exists when the conventional 
measuring method using a Boley gauge is compared to a digital calliper and the latest Carestream model analysis software. The study made use of three different methods for orthodontic study model analysis and evaluated the differences between the three systems. The study was conducted by measuring five parameters on the study models of one hundred cases with all three of the instruments.

A sample t-test was conducted and the statistical analysis showed the difference between the three instruments were not statistically significant for the intermolar width $(p=0.849)$, intercanine width $(p=0.657)$, mesiodistal width of tooth $11(p=0.178)$ and mesiodistal width of tooth $41 \quad(p=0.240)$. The difference in measurements between the three instruments for the mesiodistal width of tooth 46 was statistically significant $(p<0.01)$.

Although a statistically significant difference was shown on the measurements obtained for tooth 46 , the difference was not found to have a clinical significance to the orthodontist when conducting a model analysis. The results of the five measurements for each of the three instruments were accurate and similar when compared to one another. An average value of $40.5 \mathrm{~mm}$ (intermolar width), $34.3 \mathrm{~mm}$ (intercanine width), $8.5 \mathrm{~mm}$ (mesiodistal width of tooth 11), $10.8 \mathrm{~mm}$ (mesiodistal width of tooth 46) and $5.4 \mathrm{~mm}$ (mesiodistal width of tooth 41) was found when the values of all three measuring tools were considered, see Figure 6.

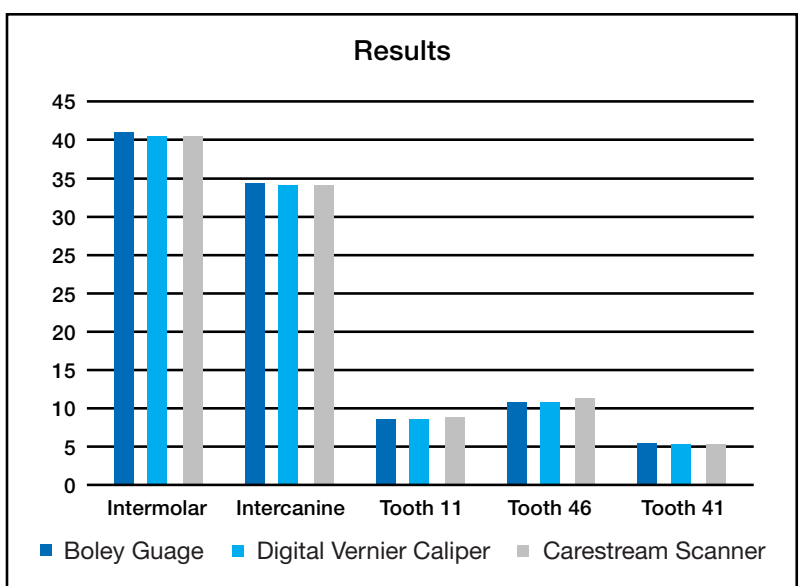

Figure 6. Results of all 5 measurements produced by the three instruments.

The values of the three instruments used in the study were very accurate which emphasizes that all three instruments are reliable and reproducible. Of the three instruments used, the digital vernier calliper took the

\begin{tabular}{|c|c|c|c|c|}
\hline \multicolumn{5}{|c|}{ Intermolar Width } \\
\hline \multirow{2}{*}{ Instruments } & \multirow{2}{*}{ Mean } & \multirow{2}{*}{ Std. Error } & \multicolumn{2}{|c|}{ 95\% Confidence Interval } \\
\hline & & & Lower Bound & Upper Bound \\
\hline 1. Boley & $40.597^{a}$ & .303 & 40.000 & 41.194 \\
\hline 2. Vernier & $40.383^{a}$ & .303 & 39.786 & 40.980 \\
\hline 3. Carestream & $40.386^{a}$ & .303 & 39.789 & 40.983 \\
\hline
\end{tabular}

\begin{tabular}{|c|c|c|c|c|}
\hline \multicolumn{5}{|c|}{ Intercanine Width } \\
\hline \multirow{2}{*}{ Instruments } & \multirow{2}{*}{ Mean } & \multirow{2}{*}{ Std. Error } & \multicolumn{2}{|c|}{$95 \%$ Confidence Interval } \\
\hline & & & Lower Bound & Upper Bound \\
\hline 1. Boley & $34.450^{\mathrm{a}}$ & .213 & 34.030 & 34.870 \\
\hline 2. Vernier & $34.196^{\mathrm{a}}$ & .213 & 33.776 & 34.616 \\
\hline 3. Carestream & $34.228^{a}$ & .213 & 33.808 & 34.648 \\
\hline
\end{tabular}

\begin{tabular}{|c|c|c|c|c|}
\hline \multicolumn{5}{|c|}{ Tooth 11} \\
\hline \multirow{2}{*}{ Instruments } & \multirow{2}{*}{ Mean } & \multirow{2}{*}{ Std. Error } & \multicolumn{2}{|c|}{ 95\% Confidence Interval } \\
\hline & & & Lower Bound & Upper Bound \\
\hline 1. Boley & $8.535^{\mathrm{a}}$ & .059 & 8.418 & 8.652 \\
\hline 2. Vernier & $8.467^{a}$ & .059 & 8.350 & 8.584 \\
\hline 3. Carestream & $8.623^{a}$ & .059 & 8.506 & 8.740 \\
\hline
\end{tabular}

\begin{tabular}{|c|c|c|c|c|}
\hline \multicolumn{5}{|c|}{ Tooth 46} \\
\hline \multirow{2}{*}{ Instruments } & \multirow{2}{*}{ Mean } & \multirow{2}{*}{ Std. Error } & \multicolumn{2}{|c|}{$95 \%$ Confidence Interval } \\
\hline & & & Lower Bound & Upper Bound \\
\hline 1. Boley & 10.779 & .059 & 10.663 & 10.895 \\
\hline 2. Vernier & 10.708 & .059 & 10.592 & 10.824 \\
\hline 3. Carestream & 11.008 & .059 & 10.892 & 11.124 \\
\hline
\end{tabular}

\begin{tabular}{|c|c|c|c|c|}
\hline \multicolumn{5}{|c|}{ Tooth 41} \\
\hline \multirow{2}{*}{ Instruments } & \multirow{2}{*}{ Mean } & \multirow{2}{*}{ Std. Error } & \multicolumn{2}{|c|}{ 95\% Confidence Interval } \\
\hline & & & Lower Bound & Upper Bound \\
\hline 1. Boley & $5.411^{\mathrm{a}}$ & .037 & 5.338 & 5.484 \\
\hline 2. Vernier & $5.333^{a}$ & .037 & 5.260 & 5.406 \\
\hline 3. Carestream & $5.409^{a}$ & .037 & 5.336 & 5.482 \\
\hline
\end{tabular}


least amount of time during the actual measuring. According to the study done by Quimby et al. ${ }^{17}$ in 2004 , the reliability of digital models are clinically acceptable

when using OrthoCAD software. A different study done by Stevens et al. ${ }^{3}$, in 2004 showed that the use of digital models would not cause an orthodontist to make a different diagnosis and can be used in model analysis and treatment planning.

Although a different software system was used in this study, the present study's results support the findings of Quimby et al. ${ }^{17}$ and Stevens et $a l .{ }^{3}$ regarding the accuracy and reliability of digital orthodontic study models and accompanyingsoftware for model analysis and treatment planning.

A study by Zilberman ${ }^{12}$ showed the use of digital callipers to have the highest accuracy and reproducibility when compared to OrthoCAD software. The present study found that all three instruments used showed the same amount of accuracy and reproducibility but that the time spent doing the measurements were faster when using the digital vernier calliper.

According to Asquith et al. ${ }^{6}$, digital models can potentially eliminate the requirement for conventional plaster models should cost not be a factor. The present study supports these findings and agrees that digital orthodontic study models can and most probably will replace conventional plaster models in the future.

According to the previous studies mentioned and this study, all three instruments will provide an accurate and reproducible result. The difference between the three comes in when cost, storage and time consumption are compared.

Both the Boley gauge and the digital vernier calliper are very affordable when compared to the intra-oral scanner and software. The scanner, however, can be used directly in the patient's mouth without the need for alginate impressions. When storage is considered the use of the intra-oral scanner and digital models are superior.

\section{CONCLUSION}

- Parameters on conventional plaster models and digital models can be reliably measured using a Boley gauge, digital vernier calliper or Carestream software.

- All three of the instruments produced accurate measurements and can be used confidently when doing a comprehensive study model analysis for orthodontic diagnosis and treatment planning.

- A specialist orthodontist choosing one of these instruments should only compare the cost, time and storage differences since the accuracy of the three instruments are the same.

- The current advantages and future possibilities of digital orthodontic models should make them the new gold standard for model analysis.

\section{References}

1. Binder RE, Cohen SM. Clinical evaluation of tooth-size discrepancy. Journal of Clinical Orthodontics. 1998; 32: 544-6.

2. Sheridan JJ. The reader's corner. Journal of Clinical Orthodontics. 2000; 34: 593-7.

3. Stevens DR, Flores-Mir C, Nebbe B, Raboud DW, Heo G, Major PW. Validity, reliability, and reproducibility of plaster vs. digital study models: comparison of peer assessment rating and Bolton analysis and their constituent measurements. American Journal of Orthodontics and Dentofacial Orthopedics. 2006; 129: 794-803.

4. Quimby ML, Vig KW, Rashid RG, Firestone AR. The accuracy and reliability of measurements made on computer- based digital models. The Angle Orthodontist. 2004; 74: 298-303.

5. Shellhart WC, Lange DW, Kluemper GT, Hicks EP, Kaplan AL. Reliability of the Bolton tooth-size analysis when applied to crowded dentitions. The Angle Orthodontist. 1995; 65: 327-34.

6. Asquith J, Gillgrass T, Mossey P. Three-dimensional imaging of orthodontic models: a pilot study. European Journal of Orthodontics. 2007; 29: 517-22.

7. Proffit WR, Fields HW. Contemporary Orthodontics, $3^{\text {rd }}$ ed. St. Louis: Mosby, 2000: 165-170.

8. Begole EA, Cleall JF, Gorny HC. A computer program for the analysis of dental models. Comput Prog Biomed. 1979; 10: 261-70.

9. Rudge SJ. A Computer program for the analysis of study models. European Journal of Orthodontics. 1982; 4: 269-73.

10. Yen $\mathrm{CH}$. Computer-aided space analysis. Journal of Clinical Orthodontics. 1991; 25: 236-8.

11. Alcana T, Ceylanoglu C, Baysal B. The Relationship between Digital Model Accuracy and Time-Dependent Deformation of Alginate Impressions. The Angle Orthodontist. 2009; 79(1): 30-6.

12. Zilberman O, Huggare JAV, Parikakis KA. Evaluation of the validity of tooth size and arch width measurements using conventional and three-dimensional virtual orthodontic models. The Angle Orthodontist. 2003; 73: 301-6.

13. Garino F, Garino GB. Comparison of dental arch measurements between stone and digital casts. World Journal of Orthodontics. 2002; 3: 250-4.

14. Tomasetti JJ, Taloumis LJ, Denny JM, Fischer JR. A comparison of 3 computerized Bolton tooth-size analyses with a commonly used method. The Angle Orthodontist. 2001; 71: 351-7.

15. Hirogaki $Y$, Sohmura T, Satoh H, Takahashi J, Takada K. Complete 3-D reconstruction of dental cast shape using perceptual grouping. IEEE Transactions on Medical Imaging. 2001; 20: 1093-101.

16. Santoro M, Galkin S, Teredesai M, Nicolay OF, Cangialosi TJ. Comparison of measurements made on digital and plaster models. American Journal of Orthodontics and Dentofacial Orthopedics. 2003; 124: 101-5

17. Quimby ML, Vig KWL, Rashid RG, Firestone AR. The accuracy and reliability of measurements made on computer-based digital models. The Angle Orthodontist. 2004; 74: 298-303. 